\title{
Epidemiology, evaluation and management of tinea pedis
}

\author{
Ohoud Adel Turkistani ${ }^{1 *}$, Abdullah Ali Aljalfan², Meshal Mohammed Albaqami ${ }^{3}$, \\ Mohammad Mubarak Alajmi ${ }^{4}$, Abdullaziz Mohammed Bahayan ${ }^{5}$, Anwar Ajlan Alqurashi ${ }^{6}$, \\ Renad Mutlaq Alhanaki ${ }^{7}$, Fatema Fahad Alkhalfan ${ }^{8}$, Azzam Meteb Alsharif', \\ Ali Hassan Alkhabbaz ${ }^{10}$, Amal Abdulrahman Alsowaidi ${ }^{11}$
}

\author{
${ }^{1}$ Department of Family Medicine, University Medical Services Center, Jeddah, Saudi Arabia \\ ${ }^{2}$ College of Medicine, Imam Mohammad Ibn Saud Islamic University, Riyadh, Saudi Arabia \\ ${ }^{3}$ Alhusseiniya Primary Healthcare Center, Ministry of Health, Mecca, Saudi Arabia \\ ${ }^{4}$ College of Medicine, Jordan University of Science and Technology, Irbid, Jordan \\ ${ }^{5}$ College of Medicine, Alfaisal University, Riyadh, Saudi Arabia \\ ${ }^{6}$ Primary Healthcare, Ministry of Health, Mecca, Saudi Arabia \\ ${ }^{7}$ College of Medicine, Dar Al Uloom University, Riyadh, Saudi Arabia \\ ${ }^{8}$ College of Medicine, Arabian Gulf University, Manama, Bahrain \\ ${ }^{9}$ College of Medicine, King Abdulaziz University, Jeddah, Saudi Arabia \\ ${ }^{10}$ College of Medicine, Medical University of Gdańsk, Gdańsk, Poland \\ ${ }^{11}$ College of Medicine, Ibn Sina National College, Jeddah, Saudi Arabia
}

Received: 18 November 2021

Revised: 03 December 2021

Accepted: 04 December 2021

\section{*Correspondence:}

Dr. Ohoud Adel Turkistani,

E-mail: ohoudturkistani11@hotmail.com

Copyright: ( ) the author(s), publisher and licensee Medip Academy. This is an open-access article distributed under the terms of the Creative Commons Attribution Non-Commercial License, which permits unrestricted non-commercial use, distribution, and reproduction in any medium, provided the original work is properly cited.

\begin{abstract}
Tinea pedis, known as athletics foot, is defined as a dermatophyte infection leading to a condition called dermatophytosis. Usually, the mode of infection is fungal. Trichophyton rubrum is the most common organism which is responsible for the infection. This fungus is endemic in some geographical regions as in Asia and Africa. The mode of transmission and risk factors depends on several factors: the weather, type of clothes and shoes, body response to different organisms, present history, family history, and endemic geographical areas. Increased temperature and humidity were correlated in the literature to the increased incidence and prevalence of tinea pedis compared to those areas which have low temperature, wearing specific types of shoes or clothes might be associated with an increased rate of infection, especially if the shoes are adherent to the foot and occlusive, prolonged exposure to humidity and water was also shown to be among the causes for tinea pedis infections. The clinical presentation of tinea pods varies according to the site and severity of infection. Generally, antifungal drugs are effective in most cases. The application of antifungals may be in oral form or local form, or mixed form. Terbinafine was proven to be effective in mild cases to fully treat the infection within a period of one week, extending to four weeks in more aggressive cases. This was a brief look at the article. This article aimed to review tinea pedis from different prospections clinically.
\end{abstract}

Keywords: Tinea pedis, Epidemiology, Clinical picture

\section{INTRODUCTION}

Tinea pedis, known as athletics foot, is defined as a dermatophyte infection leading to a condition called dermatophytosis. ${ }^{1}$ Usually, the mode of infection is fungal.
Trichophyton rubrum is the most common organism which is responsible for the infection. This fungus is endemic in some geographical regions, as in Asia and Africa. ${ }^{1}$ However, the infecting organism can be found within the Americans or Europe, but it is usually rare 
compared to the African and Asian regions. ${ }^{2}$ The mode of transmission and risk factors depends on several factors: the weather, type of clothes and shoes, body response to different organisms, present history, family history, and endemic geographical areas. Increased temperature and humidity were correlated in the literature to the increased incidence and prevalence of tinea pedis compared to those areas which have low temperature, wearing specific types of shoes or clothes might be associated with an increased rate of infection, especially if the shoes are adherent to the foot and occlusive, prolonged exposure to humidity and water was also shown to be among the causes for tinea pedis infections. ${ }^{3}$ The pathophysiology of infection is complex. However, most of the literature claims that the primary cause of inducing the tinea pedis pathobiological changes is prolonged occlusion between toes with increased humidity, with the tight junctions in cases of wearing adherent shoes and prolonged working out for several hours. This induces the bacterial flora to colonize the skin and causes penetration and implementation with the skin epithelial layers. The primarily responsible fungus for the infection is the Trichophyton rubrum. However, the different fungus may be associated with trichophyton interdigital and Epidermophyton floccosum. ${ }^{4}$ As soon as infection penetrates the skin, the different types of fungus start releasing some mediators such as keratinase, which desolate the epithelial keratin layers within the skin. This includes an immunological mediated antibodies reaction within the body's immune system, which by role induces local inflammation with the toes, and in advanced cases, it may be correlated with systemic manifestations. ${ }^{4}$ The epidemiology of tinea pedis is claimed to be about $10 \%$ of the overall population. ${ }^{1}$ However, some sources claim that shortly about $70 \%$ of the overall population will be infected with tinea pedis for at least one time during their life. ${ }^{5}$ Main public health issues related to tinea pedis are among personal hygiene. Therefore, we interpret that people living in rural areas and underrepresented areas may have increased prevalence compared to those living in urban cities. ${ }^{6}$ In addition to that, males were more prone to tinea pedis infections than females. This is probably due to the increased activity of the males more than the females in different countries around the world. ${ }^{7}$ This article will review the literature regarding the tinea pedis infection from further prosecutions, including the epidemiological characteristics, the evaluation, management and treatment.

\section{LITERATURE REVIEW}

This literature review is based on an extensive literature search in Medline, Cochrane, and EMBASE databases which was performed on $25^{\text {th }}$ October 2021 using the medical subject headings (MeSH) or a combination of all possible related terms, according to the database. To avoid missing potential studies, a further manual search for papers was done through Google Scholar while the reference lists of the initially included papers. Papers discussing epidemiology, evaluation and management of tinea pedis were screened for useful information. No limitations were posed on date, language, age of participants, or publication type.

\section{DISCUSSION}

\section{Epidemiological characteristics}

Unfortunately, most of the studies reporting the epidemiological characteristics of tinea pedis have been since a long time ago. However, this does not deny its reliability. Several studies conducted different trials to examine the correlation between tinea pedis and different associated factors such as race, gender, age, and geographical status regarding the epidemiology and distribution around the world. ${ }^{5,8,9}$ Some studies correlated between the age and incidence of occurring. The age group between 30 up to 60 years old was more associated with tenia pedis than younger ones. Also, the incidence of infections in children was reported to be rare. ${ }^{3}$ The presentation of these results can be explained due to different theories and possibilities. At first, with the increased age, the status of immunological responses starts to decrease compared to adults and children. The children, teenagers, and adults age groups are in a developmental stage with an increased defensive system against bacterial and fungal infections compared to those older; therefore, this correlated with being one of the possible causes. ${ }^{10}$ It was found that the tinea pedis was significantly higher in certain groups of people who are occupying several jobs which require physical activity for long hours. For example, the prevalence of tinea pedis in miners was found to be an average of $75 \%$, army soldiers had an average of $60 \%$, and marathon runners had an average of $31 \%$; and also, it was found that males are more infected compared to females. ${ }^{11,12}$

Regarding geographical distribution differences, countries with cold weather and decreased overall activity of the people were found to have a lower incidence than people who live in hot and warm countries and increased physical activity. For example, African populations had 50\% higher chances of developing tinea pedis than people living in the United States of America or European countries. ${ }^{2}$ Some studies associated the risk of diabetes mellitus patients and the development of athletics foot. A study by Legge et al reported that the likelihood of developing tinea pedis in patients with diabetes mellitus was $42.5 \%$ compared to $37.5 \%$ in non-diabetic patients. However, the results were not statistically significant. ${ }^{13}$

A study by Toukabri et al reported the epidemiological profile of tinea pedis in Tunisia. ${ }^{14}$ The authors concluded that the incidence and prevalence of the infection were positively correlated with several factors with the sample of the study, including the lifestyle. People who had a healthy lifestyle had much lower infection rates than those suffering from sedentary dietary habits. Age: The age group between 31 and 60 had the highest likelihood of developing tinea pedis; the highest concentration was found at the 41 up to 50 years old group. In contrast, the 
lowest incidence was found in the children group, ranging from newborns up to ten years old. Patients with a present history of associated diseases and comorbidities were found to have higher infection rates than healthy people. Therefore, the study concluded that factors that control lifestyle quality are correlated with developing spontaneous tinea pedis infections.

\section{Clinical manifestations}

\section{General manifestations}

Tinea pedis may be present in four different forms. The first type is the interdigital type, which is the commonest; other types include vesicular, moccasin, and acute ulcerative. The clinical presentation of tinea pods varies according to the site and severity of infection. However, there are several common presentations among all types, such as pruritus and foul odor. Macerated skin is the commonest between the lateral third and fourth interdigital web spaces. ${ }^{4}$ The second most common type of tinea pedis is moccasin. Clinical presentation of moccasin type includes dry scales on toes and fissures on the plantar side of the foot; in some cases, the clinical presentation might be asymptomatic. Vesicular type of tinea pedis is associated with erythema, pain, and pus arising from the foot. Usually, the vesicular type is rapid and causes severe infection compared to the other previous types. The ulcerative tinea pedis usually results from secondary bacterial infections, causing erosions and lacerations of the skin between the interdigital web spaces. Therefore, sometimes the ulcerative type of tinea pedis is called the chronic exacerbation of the interdigital type; since this type commonly affects the interdigital spaces but with a more advanced clinical presentation, however, despite all the different types of tinea pedis. The dorsal surface of the foot does not get infected in all cases. If left untreated, the infection progression worsens, causing severe local inflammation and pain. ${ }^{15}$

\section{Pathophysiology}

The pathophysiology behind dermatophyte infections relies on arthrospores. Factors inducing arthrospores activity include the high temperate, humid weather and decreased ventilation to the foot, which by role stimulates the proliferation of fungus. The natural physiological protecting mechanism against dermatophytes is the sebum, which has an inhibitory role in the fungal proliferation of dermatophytes. ${ }^{16}$ Dermatophytes produce several enzymes such as lipase, kinase, and keratinase, which have a destructive effect on the skin layers, especially the most superficial. ${ }^{16}$ The expression pattern is induced through specific receptors. The dectin-1 receptors and toll-like receptors are the primary targets for dermatophytes that have action. The expression induced an inflammatory reaction releasing cytokines and chemotactic factors, which have a significant role in causing pain and swelling for toes. ${ }^{17}$ Chemotactic factors are mediated through the monocytes, lymphocytes, and neutrophils, producing reactive oxygen species such as hydroxyl radicals and hydrogen peroxide, the main destructor for proteins within the cell membrane and causing cellular degeneration. The activation of lymphocytes (T-lymphocytes) induces a proliferative response causing an inflammatory reaction, either local or systemic affection. ${ }^{18}$

\section{Complications}

The complications resulting from tinea pedis are variant, but almost all are local and related to the infecting organism. Cellulitis is among the most typical manifestations associated with tinea pedis in unhealthy people. However, the progression of cellulitis is not directly correlated to the dermatophyte's organisms. The link is associated with skin penetration and exposure from the fungal infection, which allows the bacterial organisms from the outside surroundings to penetrate the skin and cause secondary bacterial infections. ${ }^{19}$ It was observed that patients with altered immune status, such as in autoimmune disorders or diabetes mellitus, have a higher risk of developing secondary complications compared to the healthy person without associated comorbidities. The reason behind this is the delayed and weaker immune response in patients with previous comorbidities. ${ }^{20}$

Rarely, some cases may suffer from systemic complications such as asthma and pulmonary complications. The pathobiology behind respiratory manifestations is mainly due to excessive allergic reaction, which affects the whole body system. ${ }^{21}$ In healthy patients who do not suffer from other diseases, the most typical complications are limited to dermatological manifestations such as mild allergy and local skin rash due to the inflammatory reaction induced by the inflammatory mediators. ${ }^{22}$

\section{Evaluation and management}

The evaluation and assessment of tinea pedis are mainly clinical. The patient comes to the clinic complaining of pain in his toes, scanty foul odor, and discomfort while wearing adherent chose. Examination of the interdigital web spaces will show a greyish batch on the skin, which may feel itchy and burn while touching. However, the clinical examination in some cases might not be enough, which requires a secondary confirmatory microbiological laboratory test. ${ }^{10}$ The details of laboratory analysis include microscopic examination, including fungal culture for the specimen and microscopic examination for the skin. $\mathrm{KOH}$ examination is the study of choice to identify the fungal behavior and characteristics. ${ }^{23}$ Usually, the first line physicians for tinea pedis are family physicians or general practitioners if the diagnosis was tricky and unconfirmed. A dermatologist may be consulted for a confirmatory diagnosis. In rare, advanced cases, surgical debridement may be indicated. ${ }^{24}$

The management and treatment are dependent on the severity of the case. Generally, antifungal drugs are 
effective in most cases. The application of antifungals may be in oral form or local form, or mixed form. Terbinafine was proven to be effective in mild cases to fully treat the infection within a period of one week, extending to four weeks in more aggressive cases. ${ }^{24}$ Although the usage of an antifungal is continued until the symptoms of the infections are resolved. Combination with local drying agents such as potassium permanganate may be indicated to accelerate the process.

In cases of aggressive progression of tinea pedis into tinea unguium, the usage of corticosteroids might be indicated. Unless that, steroid therapy is not favorable. ${ }^{24}$ In patients with systemic complications associated with tinea pedis, such as diabetes mellitus and autoimmune disorders, the trajectory for treatment may differ from the standard administration of antifungal drugs. For example, if the patient with suffering from a systemic allergic reaction associated with the fungal infection. Anti-allergic drugs such as fourth-generation antihistamine drugs may be prescribed. In cases of secondary bacterial infection, local or systemic antibiotics are prescribed. ${ }^{24}$

\section{CONCLUSIONS}

Since the last decade, tinea pedis infection has increased compared to the previous decades. Unfortunately, it is predicted that tinea pedis will be more infective during the following years for two primary reasons. At first, the unhealthy routine for foot care is standard, and secondly, due to the adherent shoes, which causes lack of ventilation for the toes. Accurate clinical data, such as correct diagnosis of clinical forms of tinea pedis and reliable characterization of the underlying fungus, would improve medical personnel education and expertise in the field. Cellular tools, such as PCR implanted in the nest enhance the consistency and quality of diagnostic and patient treatment and are required to determine the source of infection. The treatment alleviates tinea pedis symptoms and reduces the chance of the infection spreading to other parts of the body, decreasing environmental and systemic issues. Even though tinea pedis is one of the most frequent infectious illnesses in humans, various methods have been attempted to manage it occasionally. More research focusing on modern tax principles in a healthcare environment would increase our present understanding of the area.

Funding: No funding sources Conflict of interest: None declared

Ethical approval: Not required

\section{REFERENCES}

1. Ilkit M, Durdu M. Tinea pedis: the etiology and global epidemiology of a common fungal infection. Crit Rev Microbiol. 2015;41(3):374-88.

2. Nigam PK, Saleh D. Tinea Pedis. In: StatPearls. Treasure Island (FL): StatPearls Publishing Copyright (C) 2021, StatPearls Publishing LLC. 2021.
3. Kaushik N, Pujalte GG, Reese ST. Superficial Fungal Infections. Prim Care. 2015;42(4):501-16.

4. Leyden JL. Tinea pedis pathophysiology and treatment. J Am Acad Dermatol. 1994;31(3 Pt 2):S31-3.

5. Oz Y, Qoraan I, Oz A, Balta I. Prevalence and epidemiology of tinea pedis and toenail onychomycosis and antifungal susceptibility of the causative agents in patients with type 2 diabetes in Turkey. Int J Dermatol. 2017;56(1):68-74.

6. Kiraz N, Metintas S, Oz Y. The prevalence of tinea pedis and tinea manuum in adults in rural areas in Turkey. Int J Environ Health Res. 2010;20(5):37986.

7. English MP, Turvey J. Studies in the epidemiology of tinea pedis. IX. Tinea pedis and erythrasma in new patients at a chiropody clinic. $\mathrm{Br}$ Med J. 1968;4(5625):228-30.

8. Lambert J, Richert B, Dezfoulian B, de la Brassinne M. Epidemiology, physiopathology and treatment of a frequent ailment: tinea pedis. Rev Med Liege. 2000;55(3):161-8.

9. Rogers D, Kilkenny M, Marks R. The descriptive epidemiology of tinea pedis in the community. Australas J Dermatol. 1996;37(4):178-84.

10. Ely JW, Rosenfeld S, Seabury Stone M. Diagnosis and management of tinea infections. Am Fam Physician. 2014;90(10):702-10.

11. Lamb L, Morgan M. Skin and soft tissue infections in the military. $\mathrm{J} \mathrm{R}$ Army Med Corps. 2013;159(3):215-23.

12. Cohen AD, Wolak A, Alkan M, Shalev R, Vardy DA. Prevalence and risk factors for tinea pedis in Israeli soldiers. Int J Dermatol. 2005;44(12):1002-5.

13. Legge BS, Grady JF, Lacey AM. The incidence of tinea pedis in diabetic versus nondiabetic patients with interdigital macerations: a prospective study. J Am Podiatr Med Assoc. 2008;98(5):353-6.

14. Toukabri N, Dhieb C, El Euch D, Rouissi M, Mokni M, Sadfi-Zouaoui N. Prevalence, Etiology, and Risk Factors of Tinea Pedis and Tinea Unguium in Tunisia. Can J Infect Dis Med Microbiol. 2017;2017:6835725.

15. Canavan TN, Elewski BE. Identifying Signs of Tinea Pedis: A Key to Understanding Clinical Variables. J Drugs Dermatol. 2015;14(10):s42-7.

16. Brooks KE, Bender JF. Tinea pedis: diagnosis and treatment. Clin Podiatr Med Surg. 1996;13(1):31-46.

17. Blutfield MS, Lohre JM, Pawich DA, Vlahovic TC. The Immunologic Response to Trichophyton Rubrum in Lower Extremity Fungal Infections. J Fungi (Basel). 2015;1(2):130-7.

18. Koga T, Shimizu A, Nakayama J. Interferon-gamma production in peripheral lymphocytes of patients with tinea pedis: comparison of patients with and without tinea unguium. Med Mycol. 2001;39(1):87-90.

19. Al Hasan M, Fitzgerald SM, Saoudian M, Krishnaswamy G. Dermatology for the practicing allergist: Tinea pedis and its complications. Clin Mol Allergy. 2004;2(1):5. 
20. Daeschlein G, Rauch L, Haase H. Influence of nutrition, common autoimmune diseases and smoking on the incidence of foot mycoses. Hautarzt. 2019;70(8):581-93.

21. Hegyi E, Hudáková G, Buchvald J, Hrabinová S, Bakos J. The specificity of mycins and their importance in the diagnosis of dermatomycoses. Allerg Asthma (Leipz). 1967;13(4):164-76.

22. Nakamura A, Uratsuji H, Yamada Y, Hashimoto K, Nozawa N, Matsumoto T. Anti-inflammatory effect of lanoconazole on 12-O-tetradecanoylphorbol-13acetate- and 2,4,6-trinitrophenyl chloride-induced skin inflammation in mice. Mycoses. 2020;63(2):189-96.
23. Ogasawara Y. Prevalence and patient's consciousness of tinea pedis and onychomycosis. Nihon Ishinkin Gakkai Zasshi. 2003;44(4):253-60.

24. Drago L, Micali G, Papini M, Piraccini BM, Veraldi S. Management of mycoses in daily practice. G Ital Dermatol Venereol. 2017;152(6):642-50.

Cite this article as: Turkistani OA, Aljalfan AA, Albaqami MM, Alajmi MM, Bahayan AM, Alqurashi AA et al. Epidemiology, evaluation and management of tinea pedis. Int J Community Med Public Health 2022;9:332-6. 